\title{
S100A4 implicated in systemic sclerosis
}

The EF-hand-containing calcium-binding protein S100A4 is "a key regulator of fibroblast activation in systemic sclerosis [SSc]," says Michal Tomcik, the lead author of a pan-European study showing that S100A4 is induced by transforming growth factor- $\beta$ (TGF- $\beta$ ) and boosts the profibrotic effects of canonical TGF- $\beta$-SMAD signalling.

SSc, a fibrotic connective tissue disease with no effective treatment options, is characterized by excessive extracellular matrix deposition by fibroblasts; a role for TGF- $\beta$ in this fibrosis is established.

The new findings from Tomcik's group, published in Annals of the Rheumatic Diseases, show that S100A4 expression is increased in the skin of patients with SSc and also position S100A4 as more than just a marker of activated fibroblasts. The analysis indicates that the protein could be a valid therapeutic drug target.

S100A4, which is known to regulate various cellular pathways and is involved in tumourigenesis, "gained attention in rheumatic autoimmune diseases such as rheumatoid arthritis and idiopathic inflammatory myopathies," says Tomcik. His research group hypothesized that it also has "a functional role in the pathogenesis of fibrotic diseases such as SSc."

\section{4 ...S100A4 expression is increased in the skin of patients with SSc ... 77}

The researchers performed arm-skin biopsies on DMARD-free glucocorticoidfree patients with SSc $(n=20 ; 12$ with limited and 8 with diffuse cutaneous disease) and healthy volunteers $(n=18)$. Levels of S100A4 protein and mRNA were higher in fibroblasts from the patients with SSc.

In vitro, either overexpression of S100A4 in fibroblasts or extracellular addition of the protein activated TGF- $\beta$ signalling pathways and increased the secretion of collagen. Conversely, siRNA knockdown of S100A4 reduced the synthesis and secretion of collagen by TGF- $\beta$-stimulated fibroblasts.

The researchers also showed that $\mathrm{S} 100 \mathrm{~A} 4^{-/-}$mice are protected from bleomycin-induced fibrosis. They measured $74 \%$ less dermal thickening and less TGF- $\beta$-SMAD signalling in the knockout mice than in wild-type control mice.

Tomcik concludes that "these findings have direct translational implications in SSc," and adds "we plan to analyse the anti-fibrotic effects of S100A4-blocking antibodies or calcimycin, an inhibitor of S100A4 transcription, in the treatment of experimental fibrosis."

Nicholas J. Bernard

Original article Tomcik, M. et al. S100A4 amplifies TGF- $\beta$-induced fibroblast activation in systemic sclerosis. Ann. Rheum. Dis. doi:10.1136/ annrheumdis-2013-204516 